\title{
Indices d'affectivité dans les corrections des enseignants et leur impact sur le rapport au texte chez les élèves
}

\author{
Sara Mazziotti ${ }^{1, *}$ \\ ${ }^{1}$ CLESTHIA (EA 7345) Université Sorbonne Nouvelle - Paris 3, France \& Università di Bologna, \\ Italie
}

\begin{abstract}
Résumé. La rédaction d'un texte implique plusieurs processus cognitifs auxquels s'ajoute également une dimension émotionnelle chez l'élèvescripteur. L'influence du contexte scolaire et l'apport de corrections de la part de l'enseignant sur le premier jet sont également des aspects à creuser lorsqu'on s'intéresse aux émotions en jeu dans la rédaction et dans la production d'un deuxième jet. Dans le cadre d'une étude plus large sur les pratiques d'interventions des enseignants en CE2 et en CM2 dans deux écoles en France et dans deux écoles en Italie, nous essayerons de mettre en relief des indices du « rapport au texte» chez les élèves, en particulier lorsqu'ils abandonnent leur premier jet en faveur d'une écriture ex novo. Sur la base d'une mise en comparaison du premier et du deuxième jet, nous observerons la fréquence de deux autres cas de figure dans les deux contextes linguistiques : la mise au propre et le remaniement plus consistant du texte.
\end{abstract}

\begin{abstract}
Several cognitive processes are involved in writing a text. In addition to that, the emotional dimension of the student-writer must also be taken into account. Both the influence of the school context and the teacher's corrections on the first draft need to be explored when considering the emotions involved in writing and in producing a second draft. In the context of a broader study on third and fifth degree teachers' intervention practices within two schools in France and two in Italy, we will try to highlight similarities in the "relationship to the text" among students, especially when they ignore their first draft to write from scratch. By comparing the first with the second draft, we will analyze the frequency of two other phenomena in both linguistic contexts: the "mise au propre" (only surface corrections) and a more important reworking of the text.
\end{abstract}

*sara.mazziotti@studio.unibo.it 


\section{1 «Prédicteurs des émotions » visibles et moins visibles en contexte scolaire}

Les copies d'élèves sont de plus en plus étudiées sous différents points de vue à partir de questionnements multiples auxquels une collaboration stricte entre des disciplines telles que la linguistique, la psycholinguistique, la didactique, l'informatique essayent de répondre. Elles portent en effet les traces de l'évolution d'un texte, produit au sein d'un système d' « attentes enseignantes en termes de norme langagière et socio-langagière » $[1, \mathrm{p}$. 45], qui témoignent de l'interaction avec l'«enseignant-correcteur» [2]. Tout particulièrement, lorsque nous comparons un premier jet de l'élève corrigé par l'enseignant et son deuxième jet, nous avons accès aux traces de « relecture-révision » [3] mises en place par l'élève après avoir reçu les corrections de l'enseignant. Quels sont les choix à l'origine du passage de la première à la deuxième version du texte de la part de l'élève et quels indices d'affectivité des corrections des enseignants pourraient influencer ces choix ?

Tout d'abord, essayons de définir la conception des émotions dans notre étude qui s'appuie sur un corpus de productions écrites françaises et italiennes de CE2 et de CM2 : lorsque nous comparons deux versions d'un texte rédigées dans le cadre d'une consigne de réécriture, nous ne pouvons pas observer « les réactions motrices (p. ex., sourire de plaisir, froncer les sourcils $[\ldots])$ ) ou « les réactions du système nerveux autonome » $[4, \mathrm{p}$. IX] de chaque scripteur. Cependant, nous disposons d'autres indices pour déduire certaines émotions des élèves que nous regrouperont ici sous la notion de « rapport au texte ».

Avec cette expression, nous nous référons à un lien que le scripteur construit avec son texte, une sorte de familiarité avec le contenu, mais aussi avec certains choix lexicaux. Cette relation profonde mais aussi délicate pourrait être altérée ou favorisée à notre avis par certains types d'interventions de l'« enseignant-correcteur» [2] sur le premier jet. Nous avançons l'hypothèse que certaines pratiques de correction des enseignants favoriseraient plus que d'autres ce lien entre l'élève et son texte ; il s'annulerait à notre avis en cas d'abandon du premier jet et d'une écriture ex novo.

Si l'émotion « signale à l'individu ce que la situation représente, pour lui, à ses yeux, à un moment donné » et se déclenche à partir de «la perception et de l'évaluation de la situation » $[5, \mathrm{p} .10]$, nous nous demandons quelle est la perception et l'interprétation de l'élève-auteur face aux corrections de l'enseignant, lorsqu'il est amené à produire une deuxième version. "Les changements" induits par l'émotion peuvent en effet être plus « discrets » [5] donc moins visibles par rapport aux « expressions faciales » [6] : ils peuvent se manifester sous forme de « pensées, émergence de souvenirs, sensations, sentiments » [5], et dans notre cas se cacher derrière certains choix avancés lors du passage au deuxième jet.

Il est important de mentionner qu'en contexte scolaire, l'émotion est relationnée à l'apprentissage de sa "régularisation » [7]: l'élève doit apprendre à se contrôler, à faire émerger sa rationalité, à " gérer les émotions en accord avec les règles sociales » [4, p. 377]. Cependant, dans une tache de réécriture, le taux de régularisation de ses émotions demeure insaisissable, puisque les indices révélateurs de ses émotions, de son rapport au texte, sont moins visibles. Nous estimons que les pratiques de correction de l'enseignant influencent sa considération en tant qu'auteur de son texte et l'amènent à choisir l'un des trois cas de figure suivants : abandon du premier jet, mise au propre ou remaniement plus consistant du contenu et de la structure du texte.

\subsection{Transcrire pour observer les indices d'affectivité}

L'identification de points communs entre les opérations d'écriture mises en place par les auteurs et celles des jeunes scripteurs qui apprennent à écrire à l'école a déplacé l'attention vers le sujet-élève [8]. Initialement dédiée aux brouillons littéraires, la génétique textuelle 
s'est en effet intéressée aux écrits scolaires, dans son objectif d'étudier le texte « perçu comme le reflet d'un cheminement intellectuel» [9, p. 2]. À la base, la même « distinction majeure $[\ldots]$ qui oppose produit à processus, texte à production de texte, écrit à activité rédactionnelle » [3, p. 85]. La transcription et l'exploitation informatique des opérations d'écriture de l'élève (ajout, suppression, remplacement) ont permis d'étudier davantage les difficultés liées au passage de l'oral à l'écrit, la mise en pratique de l'apprentissage orthographique ou l'importance des étapes précédentes et successives à la rédaction, comme la planification et la révision [10]. Quel rôle joue la rature dans la conception et dans la révision d'un texte? Est-elle perçu en tant que synonyme d'erreur par l'élève et par l'enseignant ou pas [11] ?

La transcription des opérations d'écriture et des différentes interventions des enseignants ont permis d'ailleurs d'évaluer leur impact et leur prise en compte chez l'élève. En particulier, dans une tache de réécriture, le taux de remaniement et les choix de modification du premier texte de la part de l'élève pourrait dépendre du « "niveau" de l'intervention » de l'enseignant («mot, proposition, phrase, texte »); du «type de transformation» apportée (" addition, élimination, substitution, réarrangement »); du «caractère plus ou moins "profond" des modifications » [3, p. 86]. Dans le cadre d'une étude plus large, nous nous intéresserons alors au type et à la fréquence de certaines interventions des enseignants, afin de déduire certaines émotions à l'origine du taux de remaniement du deuxième jet chez l'élève.

\section{Une étude comparative : données et méthodologie}

Nous avons proposé la consigne de rédaction «Que feras-tu quand tu seras adulte ? Raconte une de tes journées » à des classes de CE2 et de CM2 de deux écoles en Italie et de deux écoles en France et recueilli donc un corpus artificiel. Les élèves italiens et français disposaient d'une heure de temps pour rédiger leur texte et d'une heure de temps, après une semaine environ, pour le réécrire à la suite des corrections de leur enseignant. Nous étions présents en classe pour observer le bon déroulement de la consigne et assurer le travail individuel des élèves. Ils pouvaient d'ailleurs choisir d'abandonner leur premier jet en faveur d'un texte ex novo lors de la deuxième rédaction. L'objectif de l'étude étant d'une part, de décrire et de classer les pratiques d'interventions des enseignants en CE2 et en en CM2 et, d'autre part, d'analyser la prise en compte de ces corrections chez l'élève lors d'une réécriture du texte.

Pour transcrire les deux versions des manuscrits, nous nous sommes basés sur le protocole adopté par le groupe de recherche EcriScol [12]: les fichiers texte générés sont des transcriptions linéarisées qui s'opposent aux transcriptions diplomatiques pour leur spécificité de resituer davantage les « signifiants graphiques dans une ligne continue de signes » $[13$, p. 5]. Le protocole de transcription est constitué de balises qui encadrent et délimitent toutes les procédures d'écriture (suppressions, ajouts, remplacements), les renvois à la ligne et les interventions des enseignants sur le premier jet (suppressions, ajouts, remplacements, soulignements et commentaires). Une syntaxe de balisage pour ajouter la forme normée des erreurs d'orthographe est également prévue.

L'étude comparative a été effectuée à partir de sept classes françaises et de huit classes italiennes pour un total de 202 copies françaises et 306 copies italiennes. Cependant, nous nous limiterons ici à comparer les manuscrits d'une classe de CE2 française et d'une de CE2 italienne, corrigées par deux «enseignants-correcteurs" [2] très différents. Si dans un premier temps nous évoquerons des indices concernant l'influence du contexte scolaire chez l'élève et chez l'enseignant, nous nous concentrerons dans un deuxième temps sur les effets des corrections chez l'élève lors du passage du premier jet à la version définitive. 


\subsection{L'influence du contexte scolaire : langage et jugements}

Les élèves apprennent à écrire dans un contexte scolaire constitué de plusieurs acteurs qui agissent et influencent, mêmes indirectement, les pratiques scolaires. Les jeunes scripteurs apprennent à suivre les indications de l'enseignant, à comprendre ses attentes et à sélectionner un type de langage écrit, mais aussi oral, adapté à l'école. En Italie et en France, ce langage appelé scolaire (italiano scolastico), influence également les choix de correction des enseignants : en contexte italien, ils sont souvent amenés à remplacer par exemple un mot avec un autre terme plus formel, dans le but d'élever le discours de l'élève vers un registre plus soutenu et surtout de faire varier le texte (Fig. 1).

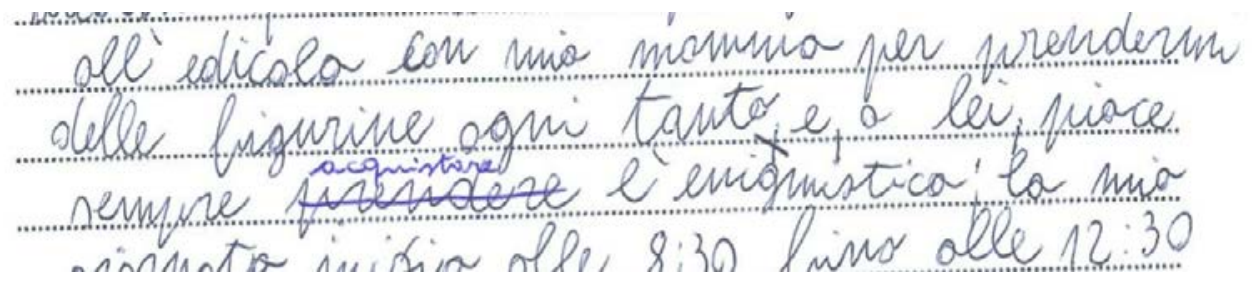

Fig. 1. Extrait d'un manuscrit d'un élève italien de CM2 (EC-CM2-2017-PAC1-D1-E1-V1) dans lequel l'enseignant remplace le verbe « prendere » (prendre, en français) avec le verbe « acquistare » (acquérir, en français).

Observons cet extrait dans lequel l'enseignant supprime le verbe « prendere » (prendre) et le remplace avec le verbe " acquistare » qui correspond d'un point de vue étymologique au verbe " acquérir », mais qui n'est pas le plus utilisé au quotidien pour véhiculer ce sens. En italien, il existe, en effet, le verbe « comprare » qui aurait très bien fonctionné ici, puisque l'élève explique dans son récit que sa mère aime acheter les mots croisés («a lei piace sempre prendere l'enigmistica »). Malgré l'absence d'erreurs d'orthographe au sein du verbe « prendere », l'enseignant choisit de barrer le choix de l'élève et de proposer un autre mot.

Si dans ce cas c'est l'italien scolaire qui déclenche la correction de l'enseignant, dans les deux contextes linguistiques ce sont plus souvent les erreurs de langue, dites « de surface » [14]: elles sont à l'origine de la plupart des interventions (remplacements, ajouts, suppressions, mais aussi soulignements et commentaires). Les erreurs d'orthographe en particulier qui renvoient à une seule norme partagée obligent les enseignants à intervenir, car cela « touche à l'identité professionnelle » et suscite une crainte de jugement de la classe, des «parents et [de] l'administration» [15]. Une copie qui comporte encore beaucoup d'erreurs d'orthographe après avoir été corrigée pourrait donner lieu à un jugement négatif du travail de correction de l'enseignant. Par conséquent, c'est justement l'aspect linguistique du texte que les élèves soigneront le plus au moment de la réécriture, puisque le plus massivement corrigé.

\section{Indices lors du recueil}

Pour constituer notre corpus de productions écrites italiennes et françaises, nous avons assisté aux deux séances de rédaction, donc nous étions présente en classe à côté de l'enseignant pour lire et soumettre la consigne. Déjà au moment du recueil, des indices émergent à propos de l'influence du contexte scolaire sur les émotions du jeune scripteur : qui lira mon texte, mon maitre ou l'observatrice? Pour en faire quoi ? À quoi il doit ressembler?

Les élèves produisent leurs textes pour l'enseignant, c'est-à-dire qu'ils s'attendent à une correction qui vise à détecter le dysfonctionnement. Tout texte scolaire subit les influences 
du contexte scolaire et de l'attente de jugement de l'enseignant. Ce contexte implique un rapport de "dominant-dominé »[16], constitué d'une duplicité de rôles occupés par l'enseignant d'une part et par les élèves d'autre part, et de la perception de la position occupée en opposition à celle de l'autre acteur.

Lors du recueil des données, l'enseignant était présent en classe au moment des deux rédactions, afin d'aider les élèves en cas de blocage, mais il n'était pas autorisé à travailler de manière collective avec eux avant leur rédaction individuelle. La présence de l'observatrice en classe a certainement contribué à altérer l'équilibre et les habitudes de chaque groupe-classe, mais était indispensable, afin de limiter au maximum le nombre de variables (déjà très consistant à cause de deux contextes linguistiques différents). Notre protocole de recueil invitait d'ailleurs les élèves à ne pas gommer ni utiliser d'effaceur, pour que les mots supprimés demeurent lisibles. Ces indications étaient inusuelles et difficiles à appliquer pour les élèves, qui avaient plutôt la tendance à cacher leurs biffures et leurs essais orthographiques. Dans un contexte scolaire, qui les invite à bien écrire et soigner la mise en page, ils ne comprenaient pas notre intérêt vers leurs biffures, au point que nous avons été obligés de les rassurer et de répéter plusieurs fois cette consigne. Dès le début de la scolarité, c'est la page propre et bien structurée qui mérite d'être présentée à l'enseignant et parallèlement toute rature est, pour eux, symptôme d'erreur. Et cela de manière similaire chez les élèves français et chez les élèves italiens : une tendance à vouloir privilégier le crayon par exemple, puisqu'effaçable, a été dégagée dans les deux contextes linguistiques.

\section{Indices lors de la réécriture}

Entre la rédaction du premier jet et la rédaction de la version définitive, les enseignants ont apporté des annotations selon leur propre méthode de correction. Ils ne connaissaient pas les objectifs précis de notre recherche, mais ils savaient que la rédaction aurait eu lieu en deux temps (avant et après leur correction). Nous pourrions nous attendre alors à un type de corrections qui facilite le remaniement du texte de la part de l'élève et qui vise à renforcer le rapport au texte chez l'élève. Cependant, comme nous le verrons, certaines interventions prennent peu en considération l'auteur et se limitent à signaler les dysfonctionnements au sein du premier jet.

\subsection{Enseignant-correcteur et enseignant-lecteur}

Avant de présenter les résultats de la mise en comparaison des copies d'élèves corrigées par un enseignant italien et un enseignant français de CE2, nous évoquons la distinction entre le « correcteur» et le « lecteur » mise en avant par Pilorgé [2] : le premier est un enseignant plus superficiel, pris dans sa pratique corrective et amené à corriger sans se poser trop de questions; le deuxième est en revanche un enseignant attentif à laisser des traces pour montrer qu'il a véritablement lu la production de l'élève et qu'il a prêté attention au contenu. Pilorgé le décrit en soulignant que sa réaction « est en effet entièrement dirigée vers le conseil de réécriture et la suggestion de révision » et que «si une difficulté de lecture apparaît, [...] le lecteur se remet en cause et relit en supposant l'insuffisance de sa lecture première » $[2, \mathrm{p}$. 132]. L'objectif de correction est commun à l'enseignant-lecteur et à l'enseignantcorrecteur, mais le premier fait l'effort de lire le texte de l'élève selon un « contrat de lecture ordinaire » et de comprendre la logique du scripteur, alors que l'enseignant-correcteur le considère uniquement un texte qui doit d'être corrigé. C'est le rapport maitre/élève qui est encore ici très présent et qui est strictement lié à l'idée que « l'erreur de l'élève détient [...] le redoutable pouvoir de nier l'efficacité du maitre, de remette en cause un travail dont la qualité intrinsèque n'est rien si elle n'est confirmée par la réussite de celui qui apprend » $[17$, p. 39]. 
A cette opposition, Pilorgé associe dans son étude un classement de cinq postures de correction parmi lesquelles nous ne mettons en avant que le "gardien du code» et le « lecteur-naïf» [2]. Le premier, qui se focalise précisément sur l'aspect formel du texte, sur l'orthographe et la grammaire, en corrigeant souvent massivement la copie (Fig. 2), est un véritable « enseignant-correcteur ». En revanche, le deuxième, le « lecteur naïf », remet en question la «représentation du monde» de l'élève par le biais de questions ou commentaires qui devraient inviter l'élève à corriger des incohérences ou des liens logiques pas très clairs. Comme nous pouvons le déduire, il rejoint davantage la posture de 1 '« enseignant-lecteur », de laquelle nous présentons un exemple en figure 3.

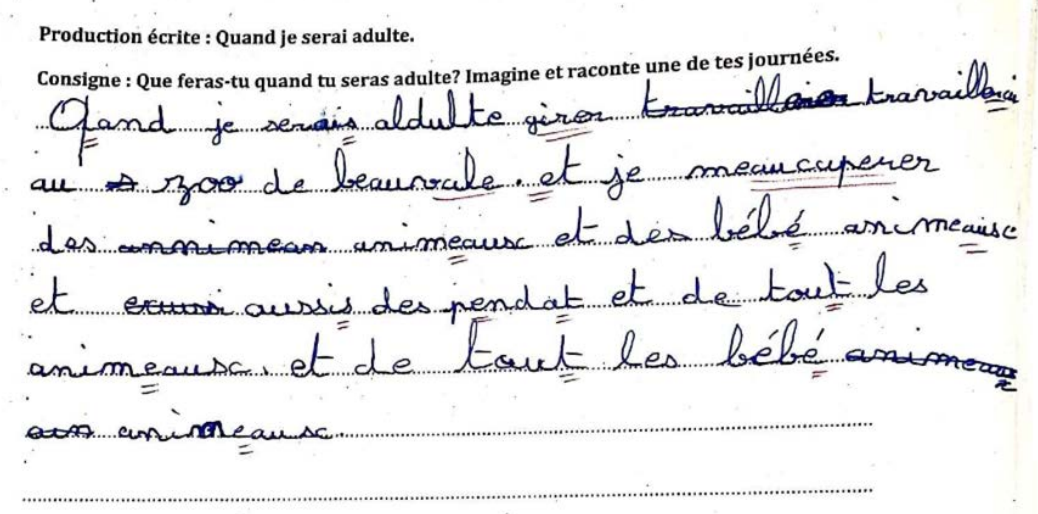

Fig. 2. Manuscrit d'un élève de CE2 français (EC-CE2-2019-COL-D1-E10-V1) dans lequel l'enseignant souligne les erreurs de langue.

Dans la figure 2, l'enseignant intervient exclusivement en soulignant en rouge les mots ou les parties du mot qui présentent une erreur d'orthographe. En comparant toutes les interventions des sept enseignants français et des huit enseignants italiens, nous avons d'ailleurs constaté que le soulignement est la pratique la plus récurrente en contexte français, mais très peu utilisée en Italie.

Aucun commentaire sur le contenu ou sur la globalité de la copie n'est apporté par cet enseignant, malgré la consigne de réécriture à laquelle l'élève devra se confronter. Nous insistons, pour conclure, sur le nombre excessif de soulignements et sur les émotions qu'ils pourraient susciter chez l'élève. Sera-t-il en mesure de trouver la forme normée de tous les mots soulignés?

Dans la figure 3, nous présentons en revanche la correction d'un enseignant italien de CE2 qui choisit de modifier les verbes au présent avec leur forme au futur. L'élève bénéficie dans ce cas d'une correction plus claire et serait amené davantage à recopier les formes corrigées dans la version définitive. L'enseignant apporte d'ailleurs en bas de page un commentaire pour réagir au contenu du récit de la jeune fille. Elle évoque deux métiers différents dans son récit et l'enseignant lui répond «Tu seras très douée dans les deux métiers !! ». Il ne s'agit pas de commentaires concernant le respect de la consigne ou la qualité du texte : il réagit au récit de l'élève dans le respect du contrat de lecture ordinaire. 


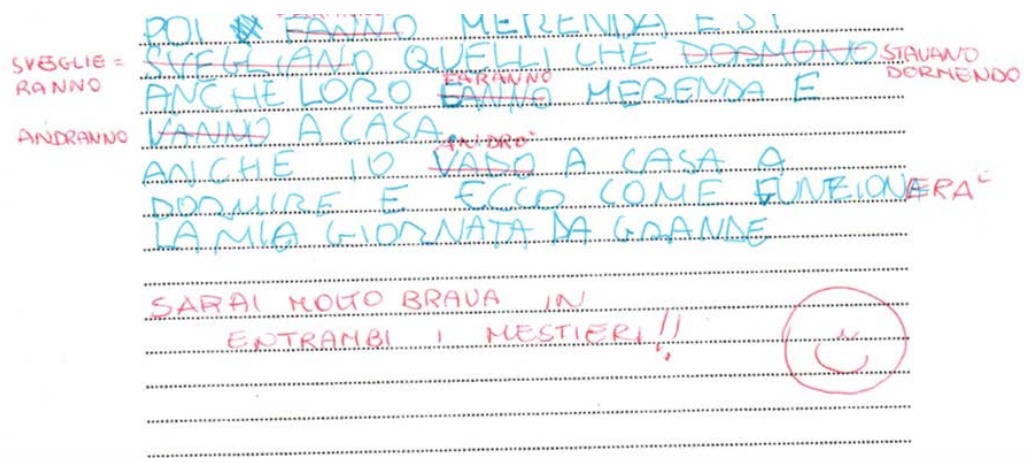

Fig. 3. Extrait d'un manuscrit d'un élève italien de CE2 (EC-CE2-2017-FOR2-D1-E2-V1), dans lequel l'enseignant ajoute un commentaire en bas de page («Tu seras très douée dans les deux métiers !! ») associé à un smiley.

Nous considérons qu'un commentaire de ce type puisse renforcer le rapport au texte que le jeune scripteur construit dès la rédaction du premier jet. Il sera donc amené davantage à ne pas abandonner son premier texte, et à le faire évoluer d'un point de vue formel ou du contenu. L'abandon du premier jet indiquerait à notre avis donc un échec dans la construction du rapport au texte : l'élève met de côté la première version, à cause probablement d'un nombre excessif de corrections reçues, qu'il devrait ensuite adopter lors de la réécriture.

\subsection{Le taux de remaniement du texte}

Intéressons-nous alors à la mise en comparaison du premier et du deuxième jet des élèves des deux classes de CE2 qui a été effectuée à l'aide du logiciel MkAlign [18]. Cet «aligneur automatique [...] est conçu pour aider l'utilisateur dans la création, l'alignement, la correction et la validation de texte » [18]. Il nous signale en rouge dans la première version à gauche tous les éléments qui disparaissent lors de la réécriture ; en bleu dans les deux versions ce qui est modifié ; en vert, en revanche, dans la version finale, ce qui a été ajouté et qui n'apparait pas dans le brouillon.

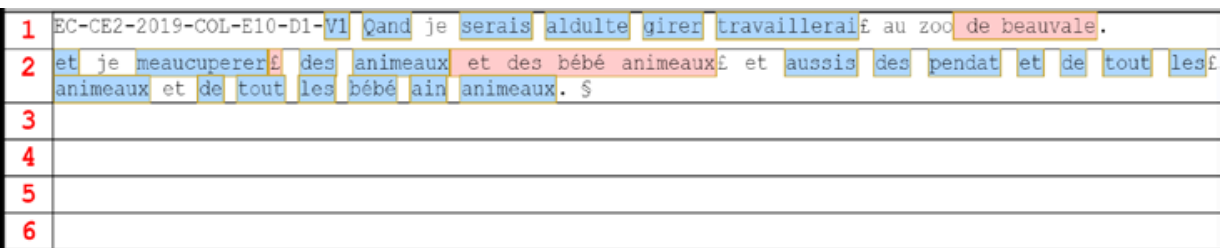

Premier jet 


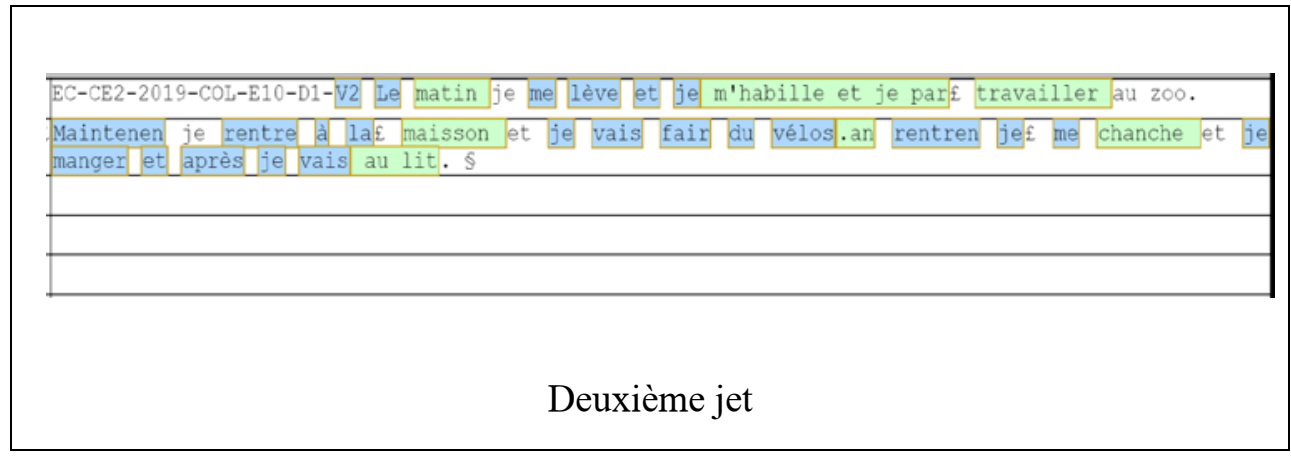

Figure 4. Alignement avec MkAlign de la mise en comparaison du premier et du deuxième jet de l'élève français de CE2, auteur du manuscrit en figure 2.

Dans la figure 4, deux captures d'écran du logiciel MkAlign nous permettent de visualiser le taux de remaniement du texte de la part de l'élève français de CE2 qui a rédigé le texte présenté en figure 2. La couleur bleue dans les deux jets indique que l'élève a produit deux textes complètement différents : il a choisi d'abandonner son premier jet et de ne pas regarder les corrections de l'enseignant (soulignements des erreurs d'orthographe, mais pas d'outils destinés à l'autocorrection). Dans l'exemple suivant, nous proposons en revanche l'alignement du manuscrit italien en figure 3 et sa version définitive.

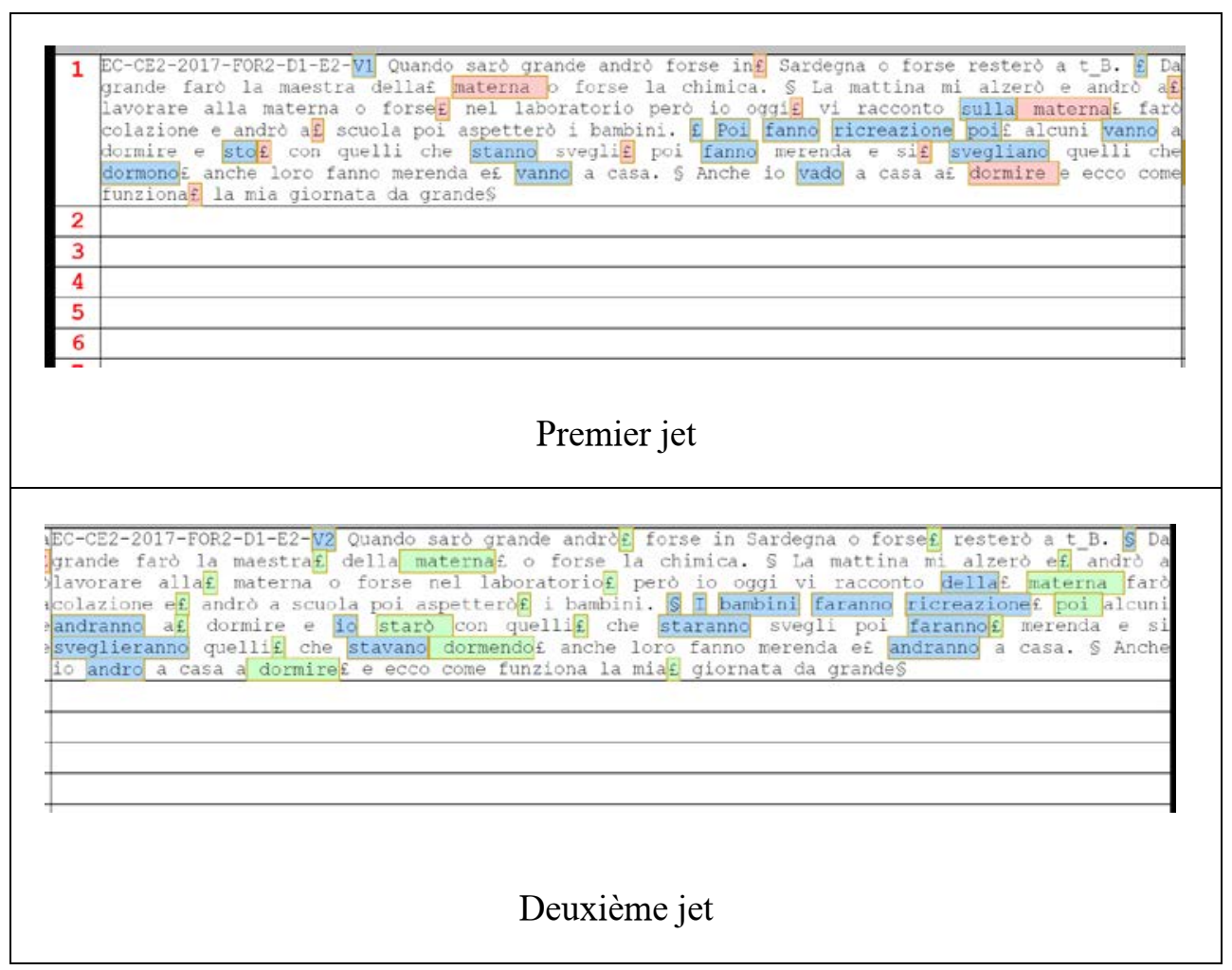

Fig. 5. Alignement avec MkAlign de la mise en comparaison du premier et du deuxième jet de l'élève italien de CE2 auteur du manuscrit en figure 3. 
Le nombre moins élevé de segments soulignés indique cette fois-ci deux textes qui se ressemblent : le jeune scripteur n'a pas abandonné le premier texte lors de la réécriture, mais il n'a pas non plus remanié de manière consistante son premier jet. Nous pouvons donc affirmer que l'élève a interprété la tache de réécriture comme une mise au propre du premier jet.

\section{Résultats}

À ces deux cas de figure, d'abandon du premier texte et de mise au propre, nous ajoutons le cas dans lequel l'élève remanie le contenu et la structure de son premier jet. Après avoir comparé les choix de réécriture des 21 élèves italiens et des 23 élèves français des deux classes de CE2, nous avons observé une différence consistante dans distribution du nombre de :

1. Abandons du premier texte en faveur d'un texte ex novo;

2. Mises au propre, donc de textes très peu remaniés et uniquement d'un point de vue orthographique ;

3. Versions plus massivement remaniées d'un point de vue du contenu, de la structure et de la présentation des idées (y compris les cas d'ajouts d'introductions et de clôtures).

\begin{tabular}{|l|l|l|l|l|}
\hline Classe & $\begin{array}{l}\text { Nombre } \\
\text { d'abandons }\end{array}$ & $\begin{array}{l}\text { Nombre de mises } \\
\text { au propre }\end{array}$ & $\begin{array}{l}\text { Nombre de versions 2 } \\
\text { remaniées }\end{array}$ \\
\hline Française & 15 & 3 & 5 & \\
\hline Italienne & 1 & 15 & 5 & \\
\hline
\end{tabular}

Fig. 6 : Tableau qui présente la répartition des trois cas de figure repérés lors de la mise en comparaison des premiers et des deuxièmes jets chez une classe française et une classe italienne de CE2.

Dans la figure 6, nous pouvons constater que le nombre de versions avec un taux de remaniement consistant est le même dans les deux contextes linguistiques ( 5 sur 21 et 5 sur 23). En revanche, à la suite du type d'intervention de l'enseignant sur leur copie, 15 élèves français sur 23 choisissent d'abandonner le premier jet, alors que 15 élèves italiens sur 21 se dédient à une mise au propre. À quoi pourrions-nous attribuer ces deux tendances opposées ?

Afin de saisir les émotions des élèves à l'origine de ces deux tendances, il nous semble approprié d'interpeller les effets des deux types d'intervention choisis par l'enseignant français et par l'enseignant italien. Le repérage et le soulignement des erreurs d'orthographe parait inhiber le travail de réécriture de l'élève français qui préfère rédiger un nouveau texte. En effet, « la détection d'un dysfonctionnement n'entraîne pas nécessairement la révision, c'est-à-dire ici la modification de certains aspects du texte. L'auteur peut ou non intervenir, peut ignorer le problème ou en différer le traitement » [2, p. 51].

Aucun élément permet à l'élève français de comprendre que l'enseignant ait véritablement lu sa production écrite : les soulignements l'invitent à proposer une nouvelle forme normée, mais ne commentent pas ses choix mis en avant lors de la rédaction de son premier jet.

En revanche, les commentaires-réactions que l'enseignant italien associent à un smiley dans toutes les copies motivent davantage l'élève à prendre en compte sa première rédaction. Son rapport au texte est maintenu, car il sait que quelqu'un a lu le contenu de son récit et il a même réagi. 


\section{Conclusions}

La pratique de réécriture des élèves à partir d'un texte corrigé nous permet de réfléchir sur certains indices d'affectivité des corrections des enseignants et évaluer le rapport que le jeune scripteur construit avec son texte. Même pendant la rédaction du premier jet, d'autres indices de ce rapport peuvent émerger en révélant l'influence du contexte scolaire chez l'élève d'un point de vue linguistique et des attentes de l'enseignant. La tendance à cacher ses ratures et à utiliser l'effaceur signale par exemple une intériorisation des indications récurrentes concernant la remise d'un texte " propre », mais aussi une inhibition du travail de révision et de réécriture déjà dans la première rédaction.

Pour les élèves réécrire signifie "recopier passivement leur devoir», proposer une « mise au net trop souvent désespérément identique au brouillon, mais propre, conforme à des règles sur lesquelles on ne s'interroge plus » [17, p. 9]. Mais qu'est-ce qui se passe quand on leur offre la possibilité d'abandonner, s'ils le souhaitent, leur premier texte et d'en produire un autre à partir de la même consigne ? En comparant le nombre d'interventions de sept enseignants français et de huit enseignants italiens, nous avons constaté dans notre étude plus large que les premiers apportent plus de corrections que les enseignants italiens [19].

Après avoir comparé en revanche les pratiques d'intervention d'un enseignant français et d'un enseignant italien de CE2, nous avons remarqué une altération du « rapport au texte » chez l'élève en contexte français, attesté par l'abandon de son premier jet. En effet, le soulignement des erreurs d'orthographe, seule pratique adoptée par l'enseignant français, a amené 15 élèves sur 23 à abandonner la première production. Les commentaires-réactions apportés en revanche par l'enseignant italien semblent motiver davantage les élèves à maintenir leur premier jet et à corriger à l'occurrence certains erreurs sur la forme ou sur le fond.

Nous concluons alors en nous demandant à quel point les enseignants prennent en compte les émotions des élèves vis-à-vis de leur texte dans un travail de production d'une deuxième version. Nous considérons qu'un exercice de réécriture et de reprise du premier texte, sans doute moins fréquent par rapport aux dictées et aux réflexions sur la langue, puisse en revanche être l'occasion pour mettre au premier plan le rapport que l'élève construit avec son texte. Cela également à travers un type de correction de la part des enseignants non focalisé uniquement sur l'orthographe et la grammaire. Il serait important de sensibiliser davantage les enseignants, d'un côté, sur l'importance de l'investissement de l'élève-auteur dans l'apprentissage de l'écriture et de l'autre côté, sur les effets que leurs interventions provoquent. Le but étant d'aller même au-delà de la « mise au propre du texte » en matière de réécriture dans les deux contextes linguistiques.

\section{Bibliographie}

1. C. Doquet, Ancrages théoriques de l'analyse génétique des textes d'élèves. Dans Boré, C. \& Calil, E. (eds), L'Ecole, l'écriture et la création. Etudes françaises et brésiliennes, 33- 53 (Académia-L'Harmattan,Louvain-la-Neuve, 2013)

2. J.-L. Pilorgé, Un lieu de tension entre posture de lecteur et posture de correcteur : les traces des enseignants de français sur les copies des élèves, thèse de doctorat (Université de Rennes, 2008)

3. M. Fayol, J.-É. Gombert, Le retour de l'auteur sur son texte : bilan provisoire des recherches psycholinguistiques, Repères pour la rénovation de l'enseignement du français, 73, 85-95 (1987)

4. D. Sander, K. R. Scherer, Traité de psychologie des émotions (Dunod, Paris, 2009) 
5. F. Cuisinier, Émotions et apprentissages scolaires : quelles pistes pour la formation des enseignants?, Recherche et formation 81 (2016)

6. P. Ekman, E. R., Sorenson, \& W. V. Friesen, Pan-cultural elements in facial displays of emotion. Science, 164, (3875), 86-88 (1969)

7. Frijda, N. H., \& Mesquita, B. The analysis of emotions: Dimensions of variation. Dans M. F. Mascolo \& S. Griffin (eds.), Emotions, personality, and psychotherapy. What develops in emotional development? 273-295. (Plenum Press, New York,1998)

8. C. Fabre, Les Brouillons d'écoliers ou l'entrée dans l'écriture. (L'atelier du texte, Grenoble, 1990)

9. C. Doquet, Etude génétique de l'écriture sur traitement de texte d'élèves de Cours Moyen 2, thèse de doctorat (Université de la Sorbonne nouvelle - Paris III, 2003)

10. L. A. Teruggi, Leggere e scrivere a scuola: dalla ricerca alla didattica (Carocci editore, Roma, 2019)

11. M.-C. Penloup, La rature n'est pas un raté. Plaidoyer pour le brouillon (Académie de Rouen, 1994)

12. EcriScol : http://syled.univ-paris3.fr/ecriscol/CORPUS-TEST/

13. P.-Y. Testenoire, Transcrire des écrits scolaires : entre philologie et génétique textuelle, Corpus 16, 87-109 (2016)

14. J.-É. Gombert, Le rôle des capacités métalinguistiques dans l'acquisition de la langue écrite. Dans Repères, recherches en didactique du français langue maternelle, 143-156 (1991)

15. J.-P. Astolfi, L'erreur, un outil pour enseigner (ESF Sciences humaines, Issy-lesMoulineaux, 2015)

16. P. Bourdieu, Ce que parler veut dire, l'économie des échanges linguistiques, s.1, (Fayard, Paris, 1982)

17. O. \& J. Veslin, Corriger des copies. Évaluer pour former, (Hachette, Paris, 1992)

18. Site MkAlign (Fleury, Paris 3) : http://www.tal.univ-paris3.fr/mkAlign/\#p1

19. S. Mazziotti, Traitement automatique des interventions des enseignants en CE2 et en CM2 en France et en Italie : entre grammaire et contenu, Bellaterra Journal of Teaching \& Learning Language \& Literature, 13 (2013) 